\title{
Post-Acute Care: What Does it Have to Do with Me?
}

\author{
Connecting the Policies and Incentives on Emergency Medicine, Hospital Medicine and \\ Post-Acute Care
}

\author{
Amy E. Boutwell · Steve Silber • Danny Nguyen • \\ Lorraine Ryan $\cdot$ Laura Melville
}

Published online: 25 January 2014

(C) Springer Science+Business Media New York 2014

\begin{abstract}
Major policies in payment and regulation over the past several years have focused on improving health care in all departments and reducing avoidable emergency department (ED) visits and readmission. These new pressures on delivery of health care require collaboration among staff of emergency, hospital medicine, and postacute care departments, not just transfer of patients to each other. This article will describe policy and regulatory pressures on emergency departments, hospitals, and postacute settings, identify common areas of interdependence, and make recommendations for successful collaboration.
\end{abstract}

Keywords Affordable care act - Hospital medicine .

Post-acute care $\cdot$ Readmissions

\section{Introduction}

The cost of healthcare in the United States is high, and growing at an alarming rate. National health spending will account for nearly one-fifth of the US economy and reach $\$ 4.8$ trillion by 2021 ; almost half of the costs of health care will be incurred by federal, state, and local governments [1]. A significant force driving the high cost of health care is the prevailing fee-for-service payment system, in which

\footnotetext{
A. E. Boutwell

Collaborative Healthcare Strategies, Lexington,

MA 02420, USA

S. Silber $(\bowtie) \cdot$ D. Nguyen · L. Ryan · L. Melville

New York Methodist Hospital, 506, 6th Street, New York,

NY 11215, USA

e-mail: Sts9005@nyp.org
}

providers are rewarded for volume. Numerous studies have revealed that "more" care is not necessarily "better" care, and that, in fact, the US ranks exceptionally low on the basis of health care status as a function of what we spend as a nation [2].

A major policy focus of the Affordable Care Act is to stimulate restructured care by the delivery system to promote "better care at lower costs, through improvement." The objective of the Affordable Care Act is to reduce costs by penalizing hospitals for excessive readmissions, by creating new payment structures for periods of care, and for population management, and by transferring patients from expensive facility-based long-term care to less costly community-based care and services [3].

As the healthcare industry undergoes substantial transformation, emergency medicine physicians and hospitalists find they are crucial participants in a new environment of policies, projects, and regulations that are of great importance to other participants with different incentives. Payers want to reduce costs, organizations want to maximize profits, and providers are often expected to serve both-controlling costs while generating revenue. Even within a single organization, many factors affecting quality, cost, and strategy must be simultaneously addressed.

In this paper we attempt to describe several new areas of policy and payment incentives that require emergency medicine and hospital medicine to collaborate more closely with post-acute care departments to reduce avoidable hospitalization and readmission. Emergency medicine physicians and hospitalists making admission-discharge decisions are critical participants in delivery of the objectives not only of the Affordable Care Act but also of the hospitals and other environments in which they practice. 
Post-Acute Care: A Brief Primer for the Hospital-based Physician

Each year, more than 10 million Medicare beneficiaries are discharged from hospitals into post-acute care settings. The total cost to Medicare of care delivered by skilled nursing facilities and certified home health agencies totaled $\$ 58$ billion in 2010 [4]. These Medicare-covered services are only a small fraction of the total amount of long-term care provided, which is estimated to be over $\$ 190$ billion per year [5].

Although the sheer volume and cost of post-acute and long-term care included in these statistics is high, hospitalbased physicians typically do not have a complete understanding of post-acute and long-term care settings. Under the new pressures to reduce avoidable hospitalization from nursing facilities, and to reduce avoidable recurrent emergency department utilization and readmission, it is becoming more important for physicians to understand details of the capabilities of providers and services in nonhospital settings.

In brief, post-acute care is care provided after inpatient hospitalization. It can be thought of as short-term care, lasting less than 90 days, or long-term care, for patients requiring care for more than 90 days. Patients may move from short to longterm care, and also back to short-term (post-acute) care after a qualifying hospitalization. Both short-term and long-term care can be provided at home or in a facility.

Under Medicare fee-for-service, patients qualify for short-term post-acute care if they require skilled services, typically rehabilitative in nature, which are intended to restore a previous level of function. Rehabilitation can be further described as being acute or subacute, depending on the degree of disability and the number of hours per day a patient can actively participate in therapy. "Rehab" services can be provided at home, by Certified Home Health Agencies (CHHA) or in rehabilitation hospitals, rehabilitation units of hospitals, or skilled nursing facilities (SNFs).

Long-term care is focused on providing support for individuals who require significant assistance with the activities of daily living. This type of care may also be provided at home or in a nursing home. Medicare does not pay for ongoing long-term care, such as care required for personal care or general supervision. However, when a physician certifies that it is medically necessary to treat an illness or injury, Medicare will cover intermittent skilled nursing care, time-limited therapy, and medical social services, to help cope with the social, psychological, and medical issues that result from an illness. Medical social services in this context would include help accessing services and follow-up care, explaining how to use the healthcare system, and condition-specific education.

Payers differ in the specific care needs that are required to qualify for post-acute or long-term care. Many physicians are aware of some of the major Medicare payment criteria for post-acute care, for example the "three-day rule," which specifies that a patient must be hospitalized for three days as an inpatient to qualify for post-acute skilled nursing facility care. Emergency medicine and hospitalist physicians may not realize that a patient who has had a "three-day" stay as an inpatient can be admitted to a skilled nursing facility within 30 days of that hospitalization; it need not be a direct discharge from the hospital to the facility.

Medicare Advantage, Medicaid fee-for service, Medicaid managed care, and commercial payers have different policies regarding the conditions for which short-term and long-term care are covered. Furthermore, Medicare patients may have access to post-hospital transitional care or post-acute services via any one of several types of payment that are now available. The important thing for hospital-based physicians to remember is that post-acute coverage policy is actively evolving; services that were not available to patients yesterday may be covered as part of a new payment initiative tomorrow.

\section{New Payment Models Affecting the Hospital to Post- Acute Continuum}

The pressures that emergency medicine physicians, hospitalists, and post-acute providers feel to reduce avoidable emergency department utilization and readmission are a function of the numerous modifications to the current payment system (for example penalties) as payers attempt to introduce new payment models (for example global and bundled payments).

\section{Fee for Service Models}

Under the Affordable Care Act, the Centers for Medicare and Medicaid Services (CMS), is testing a new fee-for-service payment for post-discharge transitional care through the Community-based Care Transitions Program (CCTP) [6]. Hundreds of sites across the US are participating in the CCTP, which pays for a variety of transitional care services targeted at high-risk Medicare fee-for-service patients. In addition, CMS has approved new fee-for-service billing codes for post-discharge follow up, enabling physicians with an existing relationship with a Medicare patient to engage in telephonic and other virtual means of follow-up soon after hospital discharge; this provides incentive to ensure a face to face office visit occurs shortly thereafter [7].

\section{Bundled Payment Models}

Another large program is the CMS Bundled Payment for Care Improvement initiative [8•]. If a bundled payment is 
made, a single pre-negotiated fee is paid to an entity which then assumes the risk for a defined episode of care and is responsible for distributing the revenue among all the care providers [9]. If the care costs less than expected, the cost savings are distributed. If it costs more than expected, the entity takes a loss. The unexpected costs of an emergency department visit or hospital readmission or other complications are the financial risks incurred in this model.

\section{Shared Savings}

Sharing savings arrangements are an approach devised to counter the incentives driving over-utilization in the feefor-service payment environment. Shared savings arrangements can be very diverse, but in essence the payer offers the provider a proportion of savings achieved for a defined population. Often shared savings arrangements include quality measures to ensure that savings are not achieved at the expense of appropriate utilization and quality care [10॰]. CMS is testing shared savings through the Medicare Shared Savings Program [11].

\section{Global Payment}

Global payment models are a form of capitation in which the provider receives a global payment, on a per-patient per month basis, to provide care for a population of patients. In this model, the providers take on a financial risk for the costs associated with the care of the population. Most of these population health-risk models have quality conditions included to protect the patient from receiving inadequate care [12]. CMS's Accountable Care Organization (ACO) program, as part of the Affordable Care Act, is the federal government's foray into a global capitation demonstration project [13]. At the state level, Medicaid-managed care organizations operate within global budgets for their members.

\section{Readmission Penalties}

Many providers are now working in a market and a context that is affected by these new payment models and unrelenting performance expectations. Most of the new payment models focus on reducing avoidable hospitalizations and readmission, placing emergency medicine, hospital medicine, and post-acute providers at the center of these efforts.

\section{Medicare Hospital Readmission Reduction Program}

The "readmission penalty" for Medicare fee-for-service patients was specifically authorized by the Affordable Care Act in 2010, and went into effect on October 1 2012. The "readmission penalty" is an adjustment to a hospital's Medicare fee-for-service payments, based upon whether the hospital is found to have excess (i.e. higher than expected) readmission. In short, hospitals with higher than expected readmission for specific conditions have their payments for all hospitalizations reduced. In 2012-2013, the maximum penalty hospitals could receive was a $1 \%$ reduction in payments. In 2013-2014 the maximum penalty is $2 \%$, and the penalty rises to $3 \%$ in $2014-2015$ and beyond. The penalty was first calculated on the basis of allcause 30-day readmission after hospitalizations for acute myocardial infarction (AMI), congestive heart failure (CHF), and pneumonia (PNA). The penalties are now calculated on the basis of all-cause unplanned readmission after discharges for AMI, PNA, CHF, chronic obstructive pulmonary disease (COPD), and total hip and total knee arthroplasty. Starting in 2014, planned readmission (defined by a specific set of definitions) will be excluded from the readmission calculation for all three conditions.

Skilled Nursing Facilities Penalties for Potentially Avoidable Readmission

To align the incentives of hospital and skilled nursing facilities (SNF) to reduce unnecessary readmission, the Medicare Payment Advisory Commission (MedPAC) recommended that skilled nursing facilities also become subject to penalties for readmission. Readmission penalties for preventable hospital readmission from SNFs are scheduled to start in 2017. MedPAC also recommended that a measure be developed to address readmission that occur after discharge from SNF to home.

\section{Readmission Penalties for Medicaid}

Medicare is not the only payer implementing penalties for avoidable readmission. New York and Massachusetts are among a growing number of states that have developed readmission penalties for readmission among Medicaid patients [14, 15•]. As Medicaid expansion continues, states will increasingly shift populations into managed Medicaid plans; many plans have in place or are actively developing policies to penalize providers for higher than expected readmission.

\section{Opportunities for Emergency Medicine, Hospital Medicine, and Post-Acute Care Providers to Collaborate to Achieve Common Performance Expectations}

The rapid emergence and launch of new payment models and readmission penalties, including looming readmission 
penalties for skilled nursing facilities, places care coordination among the emergency department (ED)-hospitalpost-acute continuum as a focal point for hospital administrators and other stakeholders with the task of avoiding penalties or developing the capacity to thrive in a new payment environment (Table 1).

Although there are many means of managing hospital admission for patients in post-acute care and/or living in long-term care, it fair to say that such intervention will often concentrate on the ED. Reducing the number of transfers to an ED and reducing the number of hospital admissions from the ED are central to the numerous approaches used to reduce avoidable ED visits, avoidable hospitalization, and readmission. Fortunately, several areas of best practice exist.

\section{Shared Best Practice: Form A Cross-Continuum Team}

The Institute for Healthcare Improvement's STAAR Initiative first outlined the concept of the "cross-continuum team" as essential for bringing together providers from across the continuum to develop a strategy to achieve an inherently inter-dependent outcome, for example reduced readmission $[16 \bullet \bullet]$. The central system failure that the cross-continuum team is meant to mitigate is the lack of coordinated cross setting improvement efforts and the inability of providers in one setting to effectively understand the capabilities of providers in other settings. Emergency medicine, hospital medicine, and post-acute providers are the most frequent members of cross-continuum teams. Depending on the specific objective of your cross-setting work (i.e. avoid Medicare readmission penalties, avoid Medicaid readmission penalties, or succeed under episode-based payments for specific conditions), additional specific community-based care providers, for example community mental health and crisis teams, patient-centered medical homes, social service agencies, and elder service agencies, should be part of the effort.

Working with providers across settings to better coordinate transfers is emerging as standard best practice in hospitals across the US. Several examples of best practices and large-scale quality improvement efforts emphasize the importance of creating new working partnerships among ED, hospital, post-acute, and community based providers in "cross continuum teams," including:

STAAR Initiative (State Action on Avoidable Readmission) of the Institute for Healthcare Improvement, which

Table 1 Specific strategies ED physicians, hospitalists, and post-acute clinicians can use to collaborate

\begin{tabular}{|c|c|c|}
\hline Strategy & Comment & Reference \\
\hline $\begin{array}{l}\text { Form a cross-continuum team or } \\
\text { community coalition }\end{array}$ & $\begin{array}{l}\text { Improving transitions requires collaboration between "senders" and "receivers": } \\
\text { convene a working group comprising ED staff, hospitalists, discharge planners, } \\
\text { pharmacists, post-acute providers, and community service providers }\end{array}$ & $\begin{array}{l}\text { IHI STAAR CFMC } \\
\text { care transitions }\end{array}$ \\
\hline $\begin{array}{l}\text { Identify patient-specific risk factors } \\
\text { for readmission }\end{array}$ & $\begin{array}{l}\text { Unlike predicting readmission risk, each patient should be assessed to identify } \\
\text { issues that, if left unaddressed, could result in a readmission }\end{array}$ & BOOST 8P \\
\hline $\begin{array}{l}\text { Ensure advanced care plans and } \\
\text { objectives of care are known }\end{array}$ & $\begin{array}{l}\text { Each receiving provider in the ED, hospital, and post-acute setting should ensure } \\
\text { they receive objectives of care in transfer communications; each sending } \\
\text { provider should ensure they provide that information as part of a complete } \\
\text { transfer in care }\end{array}$ & MOLST/POLST \\
\hline $\begin{array}{l}\text { Partner with SNFs using } \\
\text { INTERACT }\end{array}$ & $\begin{array}{l}\text { INTERACT is a set of process improvements and tools directed at reducing } \\
\text { hospitalization among nursing facility residents. Collaboration of hospital-based } \\
\text { clinicians is essential to mutual success }\end{array}$ & INTERACT \\
\hline $\begin{array}{l}\text { Meaningful communication and } \\
\text { "warm transfers" }\end{array}$ & $\begin{array}{l}\text { Best practice is to develop transfer strategies and communication tools that enable } \\
\text { the "receiving" clinician to define the information needed. There is no substitute } \\
\text { for "warm transfers" between acute and post-acute providers }\end{array}$ & INTERACT \\
\hline Evaluate and return to facility & $\begin{array}{l}\text { ED providers should be aware that SNFs implementing INTERACT will indicate } \\
\text { who at the facility to call to discuss whether it is safe and appropriate to return } \\
\text { the patient to the SNF; this communication can help avert an avoidable } \\
\text { observation or inpatient admission }\end{array}$ & INTERACT \\
\hline ED care managers & $\begin{array}{l}\text { ED staff dedicated to facilitating transitions in care and optimizing referral to the } \\
\text { most appropriate care setting provide important service to ED clinicians in } \\
\text { facilitating a transition to SNF or the community, when appropriate }\end{array}$ & INTERACT \\
\hline Individualized care plans & $\begin{array}{l}\text { Emerging best practice in emergency medicine is to develop cross-setting "care } \\
\text { plans" for ED high utilizers. The input and buy in of clinicians across all settings } \\
\text { (inpatient, ED, post acute, primary care, specialty care, behavioral health, and } \\
\text { social services) is essential to making these care plans meaningful tools to reduce } \\
\text { frequent ED use and readmission }\end{array}$ & \\
\hline
\end{tabular}


was the first readmission reduction strategy to focus on a two-part strategy of improving the care process internally and improving care transitions across settings by use of a "cross-continuum team";

"Integrating Care for Populations and Communities," the "Care Transitions Aim" of the CMS QIO (Quality Improvement Organization) ninth Statement of Work, which offers a toolkit for community-coalition building, community organizing, and specific action planning steps for improving transitions across organizations and settings;

Section 3025 of the Affordable Care Act, creating the "Community-based Care Transitions Program" (CCTP) funding, which specifically required non-financially related organizations to enter into new partnerships to implement transitional care services that span settings of care;

INTERACT (Interventions to Reduce Acute Care Transfers), the evidence-based quality improvement program aimed at skilled nursing facilities to reduce avoidable hospital transfers, offering specific tools to help staff in SNFs engage with hospital partners and communicate more effectively at times to transfer to and from the hospital and ED; and

May 2013 CMS Conditions of Participation for Medicare Part A Hospitals, in which hospitals are now expected to know the capabilities of their post-acute and community base partners, among other surveyor guidance.

Shared Best Practice: Identifying- and CommunicatingReadmission Risk

Identifying patient-specific transitional care needs or risks of readmission is not the same thing as endeavoring to predict a patient's risk of readmission. Perhaps the best tool clinicians have to help prompt a thorough identification of risks of readmission is the " $8 \mathrm{P}$ " tool found in the Better Outcomes for Older Adults through Safe Transitions (BOOST) toolkit [17]. In brief, the " $8 \mathrm{P} "$ tool prompts clinicians to inquire about or identify patient-specific factors ("Ps"; for example polypharmacy, poor social support, etc.) that require attention in the transitional care plan to minimize the risk of readmission.

The observations made by hospital-based clinicians and post-acute clinicians regarding readmission risk can be captured by use of the $8 \mathrm{P}$ or other readmission risk identification instrument and subsequently shared with receiving clinicians at times of transition from one setting to another: in this way, the detailed assessments conducted in one setting can be sent to receiving clinicians across the continuum.
Shared Best Practice: Ensuring Advanced Care Plans and Objectives of Care are Known

Ensuring systematic assessment and conveyance of objectives of care is fundamental to any comprehensive effort to reduce avoidable and undesired readmission. Understanding advanced directives and family wishes and expectations of care, and continually revisiting these issues with patient and family, can prevent overly aggressive care. Several states are adopting durable cross-setting objectives in care documentation standards, variably known as POLST (Physician Orders for Life-Sustaining Treatment), or MOLST (Medical Orders for Life-Sustaining Treatment) [18].

\section{Shared Best Practice: Partner with SNFs using} INTERACT Tools

The INTERACT quality improvement program provides invaluable guidance and specific tools for post-acute facilities regarding best practices to enable early identification of changes in clinical status, communication of these observations to a clinician, and initiation of protocol-driven responses to address these changes within the facility, before initiating a transfer to the ED. Taken together as a quality improvement initiative, INTERACT has helped post-acute facilities reduce avoidable hospital transfers by $30 \%$ on average [19••].

Although many of the components of the INTERACT program are focused on improving SNF-based care to avoid a hospital transfer, equally important in the effort to improve transfers and reduce readmission for SNF patients are transfers to and from the SNF when they do occur. ED physicians and hospitalists should be aware of the enhanced communication tools that INTERACT provides to receiving ED physicians and admitting hospitalists. For example, the "Nursing Home to Hospital Transfer Form" is engineered to provide high-value, organized information to help the hospital-based physician understand the change in clinical status observed in the SNF that led to the hospital transfer. A "Nursing Home Capabilities Checklist" is also provided to hospital based physicians to help them better understand the specific clinical capabilities of the referring SNF; when safe and appropriate, these tools can help facilitate the decision to return a patient to the SNF after evaluation in the ED.

Similarly, upon hospital discharge, INTERACT has developed a "Hospital to SNF" Transfer Form and a companion tool "Hospital to SNF Data Elements" tool. These forms were developed on the basis of extensive field testing with SNFs to extract from receiving SNF clinicians the critical information they need when accepting a patient into their care. The quality improvement premise of these forms and these data elements is that the "receiving" 
clinician is the one who defines what information they need to safely and effectively receive a patient into their care.

\section{Shared Best Practice: Meaningful Communication} and "Warm Transfers"

Simple intervention, for example effective, focused, written and verbal communication strategies can reduce readmission. The INTERACT program includes a standardized hospital communication module which includes a focused reason for transfer, the contact information for the sending provider, and a statement of whether the patient can be returned to the facility after evaluation in the ED. The INTERACT packet also systematically includes documentation of objectives of care and last medication administration schedule. In addition, strategies to facilities verbal communication between post-acute and ED provider are essential when making admission decisions. Without a clear understanding of why a resident is transferred, and without clear documentation or communication of a resident's preferences, baseline status, and usual care needs, ED physicians can be inclined to admit the elderly or debilitated patient who's presenting problem is not well delineated on the assumption that the patient will be safer in the hospital rather than returned to their facility.

\section{Shared Best Practice: Evaluate and Return}

The INTERACT quality improvement program has also created a "SNF capabilities" tool that post-acute facilities can share with emergency department staff. This tool lists the specific types of nursing, diagnostic, and other services available at the facility. Use of this information in the emergency department can facilitate decisions to return the patient to the facility for ongoing care after evaluation in the ED is complete, rather than admitting the patient to the hospital.

\section{Shared Best Practice: ED Care Managers}

Because the emergency department is often followed by readmission, many successful readmission reduction efforts have involved intervention in the emergency department [16]. When a patient is identified in triage as recently discharged, an ED care manager can work with the patient, family, post-acute staff, and ED staff to clarify whether alternatives to (re)admission exist. In addition, ED care managers can develop a detailed working knowledge of post-acute facility capacities, for example on-site laboratories, radiology, and whether urgent evaluation is available on-site. These insights can assist the emergency medicine physician in determining whether it is safe to return a post-acute facility patient to the facility after evaluation in the ED, thus averting a readmission.

\section{Shared Best Practice: Individualized Care Plans}

A subpopulation of patients from long-term and post-acute settings can be expected to have subacute and chronic symptomatic manifestations of established illness. For these patients, an established plan of care should be documented and available to post-acute and emergency department staff. A common scenario from post-acute facilities is transfer for evaluation of "agitation." Many patients with dementia have outbursts that are brief and have often resolved when the patient is in the ED, where they will then stay for long periods waiting for psychological evaluation and may be hospitalized to be "stabilized" when a short break from the situation may be all that is required, or the pattern of outburst is part of the expected progress of the illness. Without a coordinated plan of care for a response to such a brief, non-pathologic change in clinical status, patients are often hospitalized when no further active medical evaluation is required.

\section{Conclusions}

New payment models and expanding penalties for readmission are driving the need for greater collaboration between hospital-based physicians and post-acute providers. Fortunately, numerous examples of best practice exist. These include improving communication and collaboration in care across settings. Improved outcomes for patients, and improved results for costs of care will result from greater coordination and collaborative decision making at times of transition from one care setting to another.

\section{Compliance with Ethics Guidelines}

Conflict of Interest Amy E. Boutwell is President of Collaborative Healthcare Strategies, and receives grants, contracts and honoraria from quality improvement groups. Steve Silber, Danny Nguyen, Lorraine Ryan and Laura Melville declare no conflicts of interest.

Human and Animal Rights and Informed Consent This article does not contain any studies with human or animal subjects performed by any of the authors.

\section{References}

Papers of particular interest, published recently, have been highlighted as:

- Of importance

- Of major importance

1. Keehan SP, Cuckler GA, Sisko AM, Madison AJ, Smith SD, Lizonitz JM, Poisal JA, Wolfe CJ. National health expenditure projections: modest annual growth until coverage expands and 
economic growth accelerates. Health Aff (Millwood). 2012; 31(7):1600-12.

2. D. Squires. Multinational Comparisons of Health Systems Data, 2012. The Commonwealth Fund, March 2013.

3. Summary of the Affordable Care Act. The Henry J. Kaiser Family Foundation. Publication (\#8061-02). http://kaiserfamily foundation.files.wordpress.com/2011/04/8061-021.pdf. Accessed 23 April 2013.

4. Medicare Payment Advisory Commission. A data book: healthcare spending and the medicare program. Washington DC: MedPAC 2011.

5. Hartman M, Martin A, McDOnnel P, Carlin A. National Health Expenditure Accounts Tram. National health Spending in 2007: slower drug spending contributes to lowest rate of overall growth since 1998. Health Aff (Millwood). 2009;28(1):246-61.

6. http://innovation.cms.gov/initiatives/CCTP/?itemID=CMS1239313. Accessed 15 Jan 2014.

7. http://www.cms.gov/Outreach-and-Education/Medicare-LearningNetwork-MLN/MLNProducts/Downloads/Transitional-CareManagement-Services-Fact-Sheet-ICN908628.pdf. Accessed 15 Jan 2014.

8. - Bundled Payments for Care Improvement (BPCI) Initiative: General Information.CMS.gov. Center for Medicare and Medicaid Services. http://innovation.cms.gov/initiatives/bundled-payments/. Accessed 5 Oct 2013. CMS home page of the Bundled Payment for Care Improvement Initiative. Contains plain-language description of the 4 models of episodes and the numerous potential conditions site may include.

9. Burns ME, Bailit M. Issue Brief No. 4.. Bundled payment across the U.S. today: status of implementations and operational findings. Issue Brief 4. 2012. Health Care Incentives Improvement Institute. http://www.hci3.org/sites/default/files/files/HCI-IssueBrief-4-2012. pdf. Accessed 3 Oct 2013.
10. • http://www.commonwealthfund.org/ /media/Files/Publications/ Issue\%20Brief/2011/Aug/1539_Bailit_key_design_elements_shared savings_ib_v2.pdf. Accessed 15 Jan 2014. A straightforward description of the basic mechanisms of shared savings with examples of the considerations given to outliers, etc. Examples are given.

11. http://www.cms.gov/Medicare/Medicare-Fee-for-Service-Payment/ sharedsavingsprogram/index.html?redirect=/sharedsavingsprogram/. Accessed 15 Jan 2014.

12. Pearce JW. The return of capitation: preparing for populationbased health care. Healthcare Financial Management Association. July 2012. http://www.hfma.org/Content.aspx?id=3234. Accessed 3 October 2013.

13. Accountable Care Organizations (ACO). 3/22/13. CMS.gov. Center for Medicare and Medicaid Services. http://www.cms.gov/ Medicare/Medicare-Fee-for-Service-Payment/ACO/index.html? redirect=/aco/. Accessed 6 Oct 2013.

14. http://www.modernhealthcare.com/article/20121005/blogs01/ 310059991. Accessed 21 Jan 2013.

15. • http://www.health.ny.gov/regulations/recently_adopted/docs/201102-23_potentially_preventable_readmissions.pdf. Accessed 15 Jan 2014. Link to the Medicaid readmission penalty policy in New York State, a leading indicator of readmission penalty policies extending to apply to Medicaid patients broadly.

16. •- www.ihi.org/staar. Accessed 15 Jan 2014. Link to the IHI STAAR Initiative, the first quality improvement recommendation to specifically work on "cross-continuum" collaboration between local providers (SNF, ED, Hospitals) to reduce readmissions.

17. www.hospitalmedicine.org/boost. Accessed 15 Jan 2014.

18. http://molst-ma.org/. Accessed 15 Jan 2014.

19. •• http://interact2.net/. Accessed 15 Jan 2014. Link to the INTERACT Initiative, the gold-standard quality improvement program targeted at reducing avoidable (ED) hospital transfers from SNFs. 